\title{
Decreased volume of hip fractures observed during COVID-19 lockdown
}

\author{
Cristina Ojeda-Thies $^{1}$ [ · Javier Cuarental-García ${ }^{1} \cdot$ Luis Rafael Ramos-Pascua $^{1,2}$
}

Received: 15 November 2020 / Accepted: 28 December 2020 / Published online: 22 January 2021

(c) European Geriatric Medicine Society 2021

\section{Key summary points}

Aim To determine if number and characteristics of hip fractures changed during COVD-19 lockdown.

Findings The socio-demographic characteristics and baseline status of patients suffering hip fractures during COVID-19 lockdown were similar, but the volume of hip fractures admitted dropped by nearly one quarter.

Message The effect of the COVID-19 pandemic fragility hip fractures may depend on the incidence of the disease itself, as well as on the severity of lockdown measures.

\begin{abstract}
Introduction The aim was to study the incidence and characteristics of fragility hip fractures admitted during COVID-19 lockdown.

Materials and methods We analysed socio-demographic and baseline characteristics of patients suffering fragility hip fractures between March 1st and May 1st 2020, period most affected by COVID-19 confinement measures. Cases $(n=64)$ were compared with controls $(n=172)$ from corresponding periods in 2018 and 2019. Poisson Generalised Linear Model regression analysis was performed to compare daily case counts, and LOESS curves compared time series.

Results No differences were found regarding age or gender distributions, pre-fracture living, ambulatory, functional or cognitive status. More patients in 2020 had a high-risk ASA score. Fewer cases [Reduction $=26 \%$ (95\% CI 1-44\%)] were admitted in 2020. The reduction seemed to correspond with confinement.

Conclusions Approximately, one quarter less fragility hip fractures were admitted to our hospital during lockdown. Patients' baseline status and socio-demographic characteristics were similar.
\end{abstract}

Keywords Hip fracture $\cdot$ COVID-19 $\cdot$ Global pandemic $\cdot$ Lockdown measures

\section{Introduction}

The coronavirus disease (COVID-19) pandemic has affected healthcare systems worldwide, including cessation of nonessential procedures and treatments in several regions. There has also been a widespread reduction in economic activity

Cristina Ojeda-Thies

cristina.ojeda@salud.madrid.org

1 Department of Traumatology and Orthopaedic Surgery, Hospital Universitario 12 de Octubre, Avda Córdoba s/n, 28041 Madrid, Spain

2 Department of Surgery, School of Medicine, Complutense University of Madrid, Madrid, Spain and mobility due to messages to stay at home as well as legally enforced lockdowns.

Orthopaedic trauma often involves surgical management. Though the volume of injuries due to recreational or workrelated activities would decrease during lockdown, this is uncertain for fragility fractures, mostly occurring indoors [1]. Hip fractures are the most representative fragility fractures, due to their associated morbidity and mortality, nearly always requiring hospital admission and surgical management [2]. While authors in Scotland [3] observed a similar rate of fragility fractures, studies from Italy reported a lower incidence of hip fractures [4, 5].

The COVID-19 pandemic has especially affected older people living in residential care, with many countries reporting outbreaks and elevated mortality rates among nursing home residents $[6,7]$. Containment measures in care 
facilities, confining residents to their rooms and cessation of group activities and visitations, could further deteriorate these already frail patients, as well as hinder supervision, increasing the risk of falls. Finally, community-dwellers suffering an injury could defer presenting to a hospital out of fear of contagion.

The goal of this study was to analyse the incidence and characteristics of hip fractures during the months most severely affected by confinement measures in Madrid, and compare them with the corresponding period of 2018-2019.

\section{Methods}

We included all patients aged 50 years or older with fragility fractures of the hip admitted to our hospital between March 1st and May 1st 2020, and compared them with data collected retrospectively from hospital records for the analogous periods during 2018 and 2019. The two preceding years were chosen to avoid spurious associations and increase statistical power. Patients were included using a prospective departmental database of all fractures presenting in the period studied, cross-checked with hospital administrative records for 2018 and 2019 using ICD-10 codes S072.0 $\times$ to S072.2x. Electronic patient records (hospital and primary care) were directly reviewed to collect data and review diagnostic codes. High-energy, periprosthetic and pathologic fractures were excluded. The methods followed were in accordance with the ethical standards of the responsible committee on human experimentation (institutional and national) and with the Helsinki Declaration. We obtained institutional review board approval, which was defined as non-interventional by the Spanish Medicines Agency.

The first death due to COVID-19 in Madrid occurred on March 3rd in a 99-year-old woman admitted from a nursing home. Phylogeographic analysis suggests the SARS-CoV-2 pathogen was likely to have been circulating in Spain by mid-February 2020 [8]. A nationwide State of Alarm was proclaimed on March 14th 2020, stopping all non-essential activities: leaving home was only permitted to go to supermarkets, pharmacies or tobacco stores, or for essential workers. People 70 years or older were not allowed to leave their homes for leisure activities until May 2nd (Table 1).

Healthcare is mainly provided in Spain through a public healthcare system managed by the regional governments, covering $98.2 \%$ of the citizens of Madrid. Each region is subdivided into basic heath zones including approximately 20.000 inhabitants, with several zones sharing a hospital for advanced medical and surgical care, including hip fracture care. Occupancy by COVID-19 cases reached 100\% of pre-existing hospital beds by March 28th, and ICU bed occupancy reached nearly $300 \%$ on April 6th [9]. The Hospital Universitario 12 de Octubre is an urban Level 1 Trauma Centre in Madrid with nearly 1300 beds and a catchment area of almost 450.000 people, approximately 75.000 65 years and older. Over two thirds of beds were occupied by patients suffering COVID-19 disease (Fig. 1), as was the case overall in Madrid [9]. No COVID-safe hospitals were defined, each centre treating patients from their established catchment areas. COVID-19 and non-COVID-19 circuits and screening of all admissions were implemented in our hospital on March 14th, 2020.

A total of 236 cases were included with an average age of 84.1 (SD 8.4) years, 64 during the lockdown period in 2020 and 172 during the corresponding period of 2018 ( 82 cases) and 2019 (90 cases).

Variables were collected as recommended by the Spanish Hip Fracture Registry [10].

We collected demographic variables (age, gender, place of residence), as well as the place of injury. We interrogated patients admitted in 2020 on the time elapsed between the time of injury and presentation to hospital. We also registered pre-fracture functional dependence in activities of daily living (Independent: Katz 5-6 points, partially dependent: Katz 3-4 points, severely dependent: Katz 0-2 points [11]), cognitive status (defining cognitive impairment as a score of more than three errors in Pfeiffer's Short Portable Mental Status Questionnaire [12]), and anaesthetic risk using the American Society of Anaesthesiologists (ASA) score [13] as an indirect measure of comorbidity. Hip fractures were
Table 1 Timeline of the COVID-19 pandemic and lockdown measures the Community of Madrid, Spain
February 25th 2020: First case confirmed in Madrid

March 3rd 2020: First death due to COVID-19 in Madrid

March 5th 2020: Restriction of visitations in long-term residential care facilities in Madrid

March 9th 2020: Closure of schools and universities in Madrid

March 14th 2020: Declaration of a national State of Alarm by the Spanish Government

March 28th-April 12th 2020: Cessation of all non-essential activity

April 13th 2020: Workers in construction and industry are allowed to return to work

April 26th 2020: Children under the age of 14 allowed to go out on short walks with one parent or another adult living in the same household

May 2nd 2020: Persons aged 70 and above allowed to go out on short walks during restricted hours

21st June 2020: Expiration of the State of Alarm 


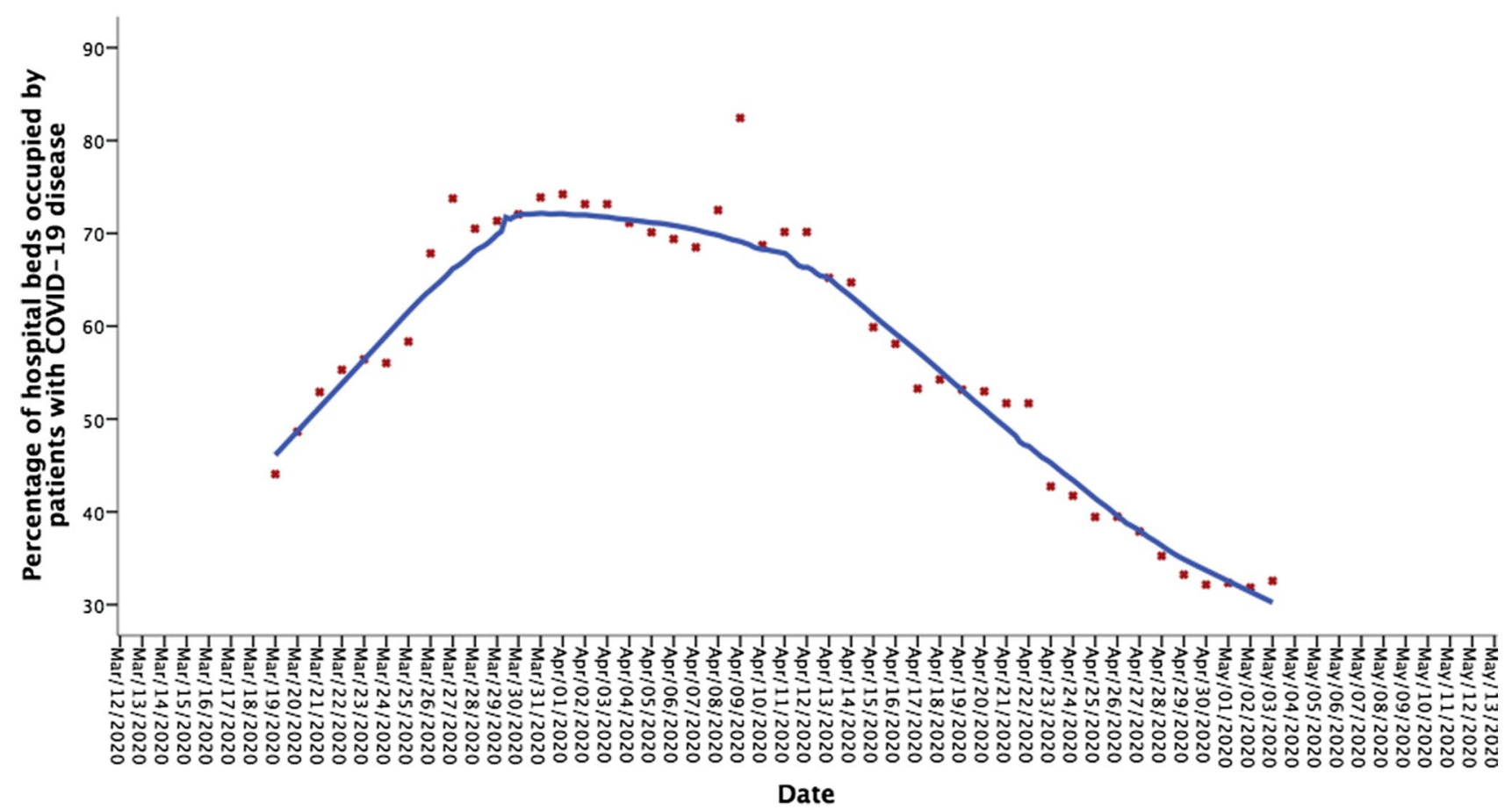

Fig. 1 Percentage of beds at our hospital occupied by patients with COVID-19 disease, between March 19th and May 3rd 2020. Markers indicate the daily percentages, the blue line shows the LOESS curve for the period observed

classified as non-displaced or Garden 1-2 intracapsular, displaced or Garden 3-4 intracapsular, intertrochanteric and subtrochanteric.

Continuous variables are reported using means and standard deviations if they showed normal distribution, and median and interquartile range if otherwise. Distributions were tested using Q-Q plots and the Kolmogorov-Smirnov test, assessing for normality and for the Poisson distribution for the count of cases presenting per day. We used Poisson log-linear Generalised Linear Models to compare the cases presenting per day in 2020 with those presenting in 2018-2019. The time series were graphically represented using daily case numbers and LOESS (locally estimated scatterplot smoothing) curves, with an Epanechnikov kernel and a smoothing parameter $\lambda=0.33$. Patient characteristics were compared using Student's $t$-test for independent samples for continuous variables, and Chi-squared and Fisher's exact tests for categorical variables. Significance was set a $p<0.05$. Data were analysed using SPSS version 20.0 (IBM, Armonk, New York, USA).

\section{Results}

The demographic and baseline characteristics of the patients treated during the periods analysed are summarised in Table 2. No differences could be observed regarding the age $(p=0.84)$ or gender distributions $(p=0.52)$. Though the proportion of patients living in residential care vs. community dwellers was similar $(p=0.38)$, less fractures occurred outdoors/in public areas $(p<0.001)$. All patients presented at the emergency department in less than 12 hours after injury, except for three patients, all of them community dwellers, who delayed presentation one, three and four days after suffering the fracture. No differences could be found regarding cohabitation of the community dwellers (alone, with spouse, with other relatives), pre-fracture ambulation, pre-fracture functional and cognitive status. A higher proportion of 2020 patients had a high-risk ASA score [ 3 or above; $p=0.01$; $\mathrm{OR}=2.80$ (95\% CI 1.19-6.59)]. Fourteen cases (21.9\%) were treated in the COVID-19 circuit, nine with a positive polymerase chain reaction (PCR) test for SARS-CoV-2 at admission, and five clinically suggestive (pneumonia, radiologic parameters, epidemiologic history). Exclusion of the patients treated in the COVID-19 circuit did not alter the differences observed in ASA risk scores $[p=0.043, \mathrm{OR}=2.5$ (95\% CI 1.01-6.32)]. No differences could be found regarding fracture type $(p=0.15)$. There was a non-significant trend towards more displaced vs. non-displaced intracapsular fractures in $2020(p=0.174)$.

The average daily number of cases admitted was 1.27 fractures (median, 1; IQR 0-2); Poisson distribution was confirmed. The Generalised Linear Model offered adequate goodness of fit (Pearson Chi-Square $=1.095)$, and showed 
Table 2 Patients' sociodemographic and baseline characteristics

\begin{tabular}{|c|c|c|c|c|}
\hline & $2018(n=82)$ & $2019(n=90)$ & $2020(n=64)$ & $\begin{array}{l}\text { Significance } \\
(2018-19 \text { vs. } \\
2020)(p)\end{array}$ \\
\hline Age, mean (SD) & $82.5(8.6)$ & $85.6(6.8)$ & $83.9(9.8)$ & 0.837 \\
\hline Gender, female (\%) & $52(63.4)$ & $62(68.9)$ & $42(65.6)$ & 0.521 \\
\hline \multicolumn{5}{|l|}{$\begin{array}{l}\text { Living situation } \\
\text { Community-dwelling }\end{array}$} \\
\hline Alone & $19(23.2)$ & $18(20.0)$ & $13(20.3)$ & 0.307 \\
\hline Spouse & $28(34.1)$ & $39(43.3)$ & $23(35.9)$ & \\
\hline Other family member & $23(28.0)$ & $24(26.7)$ & $18(28.1)$ & \\
\hline Long-term residential care & $12(14.6)$ & $8(8.9)$ & $7(10.9)$ & \\
\hline Other (religious community) & $0(0)$ & $1(1.1)$ & $3(4.7)$ & \\
\hline \multicolumn{5}{|l|}{ Place of injury } \\
\hline Private home & $51(62.2)$ & $72(80.0)$ & $54(84.4)$ & $<0.001$ \\
\hline Care facility & $12(14.6)$ & $8(8.9)$ & $9(14.1)$ & \\
\hline Public space & $19(23.2)$ & $10(11.1)$ & $1(1.6)$ & \\
\hline Cognitive impairment, Yes* $(\%)$ & $20(24.4)$ & $35(39.3)$ & $21(32.8)$ & \\
\hline \multicolumn{5}{|l|}{ Functional status $* *$} \\
\hline Independent & $48(58.5)$ & $50(55.6)$ & $32(50.0)$ & 0.479 \\
\hline Moderately dependent & $21(25.6)$ & $24(26.7)$ & $223(35.9)$ & \\
\hline Severely dependent & $13(15.9)$ & $15(16.7)$ & $9(14.1)$ & \\
\hline Unknown & & $1(1.1)$ & & \\
\hline \multicolumn{5}{|l|}{ Ambulatory status } \\
\hline Unassisted & $36(43.9)$ & $38(42.2)$ & $28(43.8)$ & 0.070 \\
\hline One cane/crutch & $34(41.5)$ & $28(31.1)$ & $30(46.3)$ & \\
\hline Two canes/walker & $7(8.5)$ & $17(18.9)$ & $1(1.6)$ & \\
\hline Unable to walk independently & $5(6.1)$ & $6(6.7)$ & $5(7.8)$ & \\
\hline Unknown & & $1(1.1)$ & & \\
\hline \multicolumn{5}{|l|}{ ASA score $(\%)$} \\
\hline 1 & $1(1.2)$ & $0(0)$ & $0(0.0)$ & 0.010 \\
\hline 2 & $20(24.4)$ & $23(25.6)$ & $7(10.9)$ & \\
\hline 3 & $43(52.4)$ & $44(48.9)$ & $40(62.5)$ & \\
\hline 4 & $18(22.0)$ & $23(25.6)$ & $17(26.6)$ & \\
\hline \multicolumn{5}{|l|}{ Fracture type (\%) } \\
\hline Intracapsular, Garden 1-2 & $6(7.3)$ & $12(13.3)$ & $1(1.6)$ & 0.145 \\
\hline Intracapsular, Garden 3-4 & $32(39.0)$ & $32(35.6)$ & $28(43.8)$ & \\
\hline Intertrochanteric & $38(46.3)$ & $42(46.7)$ & $30(46.9)$ & \\
\hline Subtrochanteric & $7(7.3)$ & $4(4.4)$ & $5(7.8)$ & \\
\hline
\end{tabular}

*Cognitive impairment defined as more than 3 errors in Pfeiffer's Short Portable Mental Status Questionnaire

**Functional status defined by Katz index of ADLs (5-6 points, independent; 3-4 points, moderately dependent; 2 points or less, severely dependent)

\begin{tabular}{lllllll}
\hline & Mean (SD) & Median (IQR) & Variance & B & Exp (B) (95\% CI) & Significance \\
\hline Intercept & & & & 0.327 & $1.387(1.195-1.611)$ & 0.000 \\
$2018-19$ (reference) & $1.39(1.22)$ & $1(0-2)$ & 1.51 & 0 & - & - \\
2020 & $1.03(1.07)$ & $1(0-2)$ & 1.14 & -0.295 & $0.744(0.599-0.992)$ & 0.044 \\
\hline
\end{tabular}

$95 \% \mathrm{CI}=95 \%$ Confidence interval 
a significant reduction in daily cases $(p=0.04)$, with $26 \%$ (95\% CI 1-44\%) less cases treated in 2020 (Table 3). The LOESS curve seems to show a correspondence of the reduced caseload with the events related to the State of Emergency (Fig. 2), with the largest decrease during the weeks confinement was enforced most (March 28th-April 12 th). The decrease was smaller than the reduction in retail and recreational activity as observed by data for the Region of Madrid from Google's Community Mobility Report [14] (Fig. 3).

\section{Discussion}

Our study showed a reduction in hip fracture incidence during lockdown due to COVID-19, though we were unable to observe any differences regarding age, gender, residential, functional or cognitive status. It is unclear what motivated this decrease. Fear of coronavirus infection could lead to patients presenting later to avoid exposure. Indeed, $5.5 \%$ of community-dwellers presented more than $24 \mathrm{~h}$ after injury. This delay could also account for the higher proportion of displaced intracapsular fractures.

Immobility affects strength, balance, and proprioception, though the degree to which older people limited mobility due to lockdown is unknown. Older persons spend most of their time with sedentary activities, especially frail subjects [15], and most injuries occur indoors. Though it is possible that immobility due to lockdowns could lead to an increase in falls and fractures, there would be a delay in this effect, which we have not observed.

The COVID-19 pandemic has disproportionally affected older people, with those institutionalised representing nearly half of deaths due to COVID-19 globally [7]. The number of hip fractures admitted from long-term care can be an indirect measure of the number of injurious falls. We have not observed any significant differences in the proportion of patients admitted from long-term care to hospital. This could

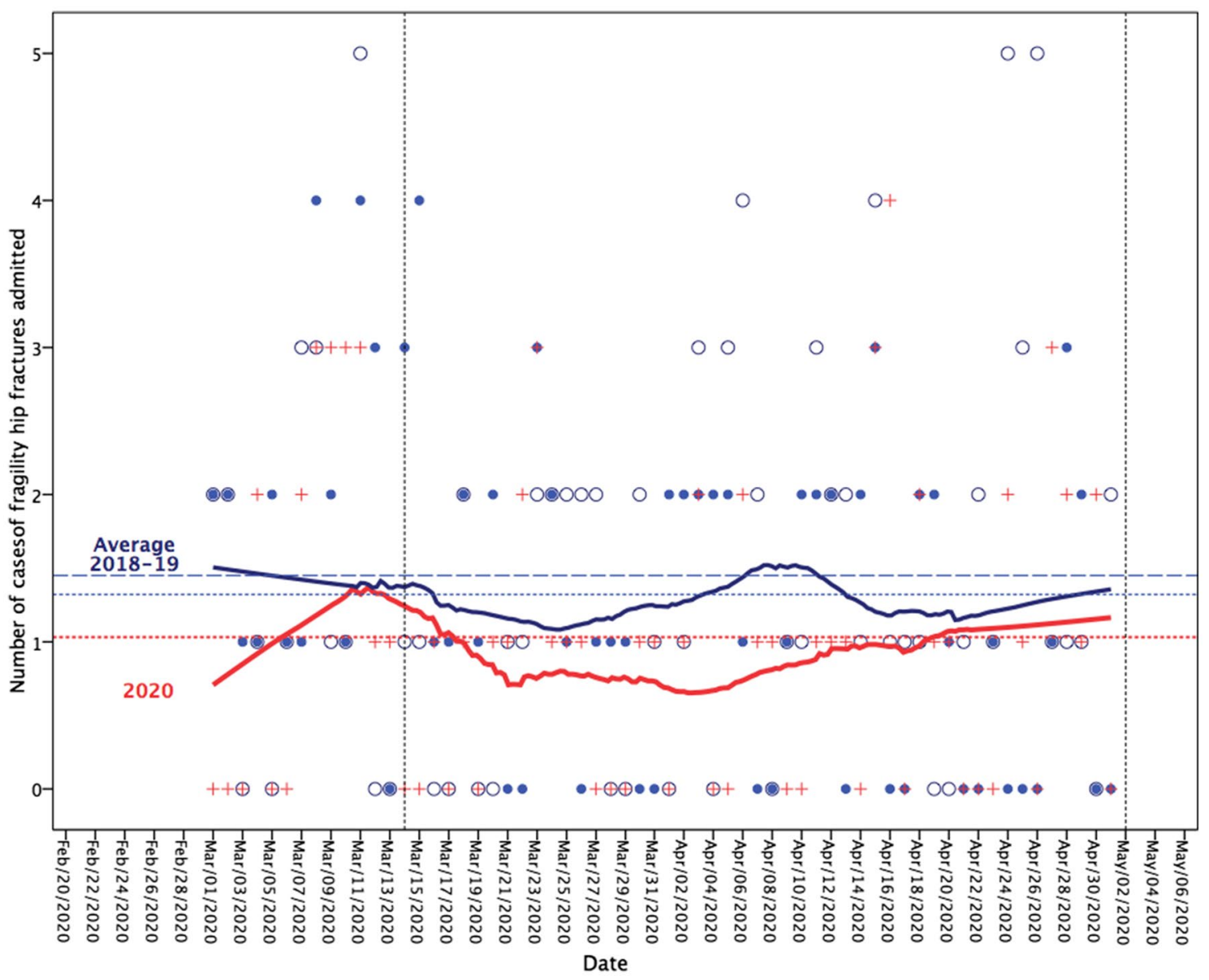

Fig. 2 Number of fragility hip fractures admitted per day during 2020 (crosses) and the corresponding periods of 2018 (solid dots) and 2019 (empty circles). Average daily case numbers for 2018 (short dotted line, blue), 2019 (long dotted line, blue) and 2020 (red dotted line).
LOESS curves for 2020 (thick, red) and the average number of cases during 2018-19 (thick, blue). The dotted vertical lines indicate the beginning and end of strict lockdown measures 
Fig. 3 Google Community mobility data [14] for retail and recreation in the Community of Madrid between mid-February and late May 2020, given as proportional variation relative to baseline status. The dotted vertical lines indicate the beginning and end of strict lockdown measures

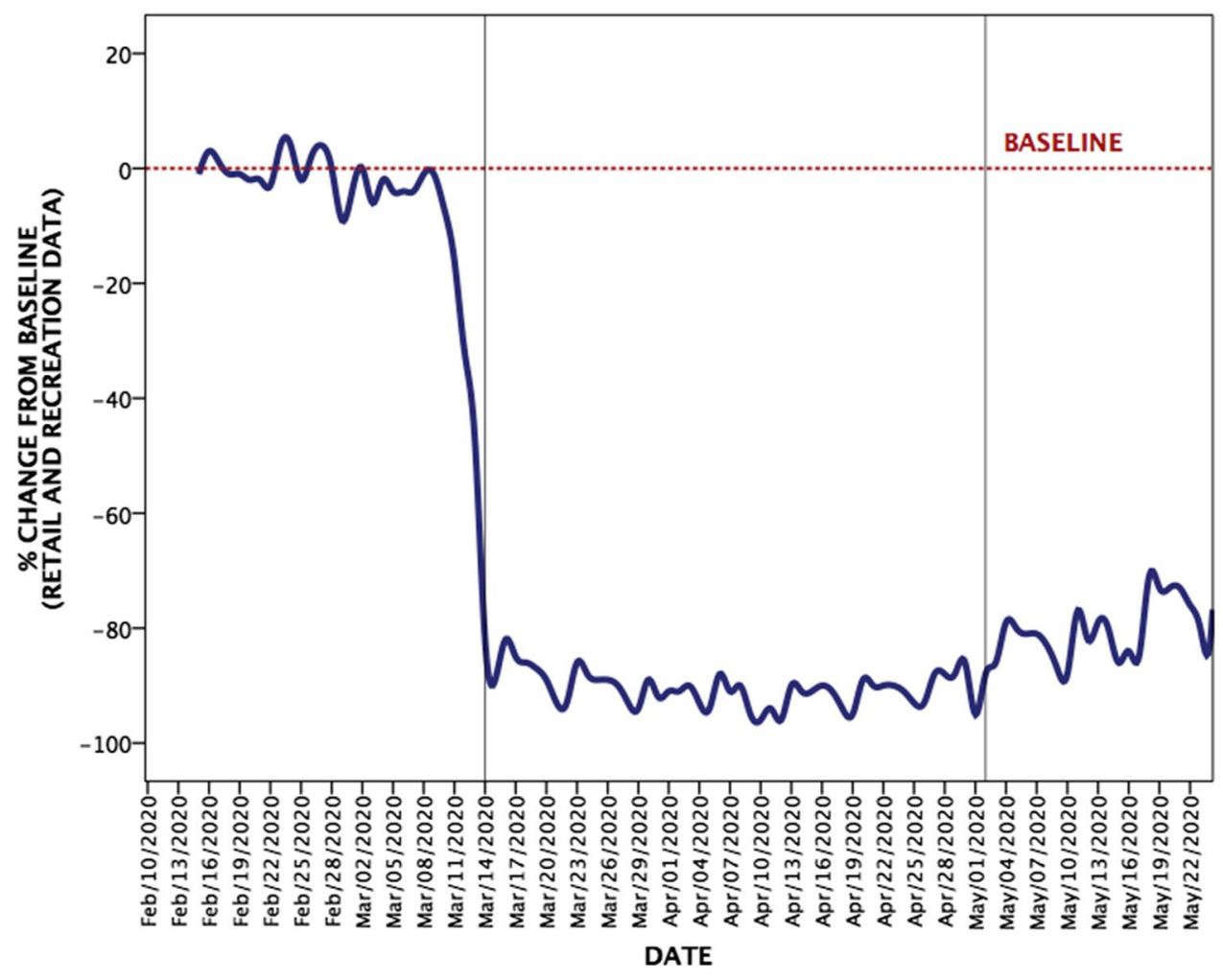

be due to there not being a significant effect, or that confinement of residents to their rooms and suspension of activities decreased the risk of falls. However, an increase of injurious falls among surviving care residents may have been offset by a decrease in the number of individuals at risk; approximately $6 \%$ of the total number of nursing care residents in Spain died during the months analysed [7]. Increased mortality in Madrid led to the use of facilities such as ice rinks as provisional morgues, so autopsies were not systematically performed. We cannot rule out that some of those deceased at home or in nursing care may have suffered an undetected hip fracture before dying.

Patients with confirmed or suspected COVID-19 disease partly justify the increased anaesthetic risk among the patients treated in 2020, but the differences remained when excluding those managed in the COVID-19 circuit. The ASA risk score is assigned during preoperative evaluation, with a certain degree of subjective interpretation. Work overload in the anaesthetic department could have led to less senior clinicians performing evaluations, erring on the side of caution when assigning a score.

Many authors have observed 22 and 50\% reductions [3-5, 16-21] in the number of trauma admissions to hospitals during lockdown, especially high-energy injuries. Park et al. [17] observed that though the overall number of referrals decreased, the likelihood of hospital admission was higher, suggesting that patients with less severe injuries would make up the largest reduction in emergency department visitations.
Thus, hip fractures, which are generally admitted to hospital, have become the most common cause of orthopaedic trauma admission and surgery during lockdown [3, 22, 23]. Though some authors have observed a decline in the number of hip fractures, others have reported similar rates compared to pre-lockdown periods. A study of 15 hospitals in Italy [4] showed that overall trauma decreased by up to $71 \% \mathrm{com}-$ pared to 2019 , with $31 \%$ less orthopaedic trauma-related hospital admissions, and surgical management of hip fractures falling up to $20 \%$. Another study from two hospitals in the Emilia-Romagna region in Northern Italy observed a $28.4 \%$ reduction [5]. Scott et al. [3] reported a $42 \%$ reduction in musculoskeletal injuries and $36 \%$ reduction in operative admissions, but the volume of fragility fractures admitted per day did not differ, as was also the case specifically for femoral fragility fractures. Similar results were observed in Barcelona, Spain, with approximately one-fourth less overall trauma admissions, but a stable number of osteoporotic hip fracture admissions throughout the periods analysed [21].

It is unclear why some have observed a reduction in fragility hip fracture admissions, while others did not. Local incidence rates and the severity of lockdown measures, as well as observation periods, may affect the results observed. The studies from Barcelona and Edinburgh observed periods of 20 and 40 days, respectively; while, the study from Northern Italy and the present study compared periods of 55 and 62 days, respectively. Finally, only $2.2 \%$ of patients with hip fracture had COVID-19 
at admission in Scotland [24], while this occurred among nearly $22 \%$ of the patients admitted to our hospital. Approximately $17 \%$ of the multicentric study from Spain by Muñoz-Vives [25] and 26\% of patients treated in Northern Italy during the first wave tested positive for SARSCoV-2 during hospitalisation [5].

Our study analyses not only the incidence of fragility hip fractures during the COVID-19 pandemic in comparison to historic controls, but also the differences in sociodemographic characteristics and baseline function. Egol compared the cases treated between February and midApril 2020 to historic controls in 2019 [26], but did not compare baseline living, cognitive or functional status, or place of injury. The Spanish healthcare system is based on geographically defined catchment areas, with a stable number of citizens assigned to a single hospital throughout time, allowing for comparison with historic controls. Data completeness is ensured due to linkage of common electronic health records throughout the entire Region of Madrid.

Our study also has several limitations. It involves a single centre, and we cannot exclude that findings could be different in other regions. The centre involved in this analysis is a major university hospital covering nearly $14 \%$ of all citizens of Madrid. Differences were observed in preoperative ASA score, which is a subjective measure. It is also possible that lockdown contributed to decompensation of baseline comorbidities. Another element subject to variation due to lockdown was the time between injury and presentation, due to several factors: first, older patients living alone but visited on a regular basis by relatives or neighbours could have fallen hours before being discovered, when otherwise they would have been found earlier. Second, patients who had suffered a fall might avoid referral to hospitals out of fear of contracting the infection. This was the case for three patients who had presented to the emergency department more than $24 \mathrm{~h}$ after falling. Due to our inability to control for this variability and to acquire data from the preceding years, we chose not to compare delay to presentation. We are unsure how to study this question, as delay to presentation to the ED is multifactorial and usually not included in registry or automated healthcare data. These data sources could, however, control for the presentation of patients to other units, and studies to elucidate the epidemiology of hip fractures during the COVID-19 pandemic on a larger scale using these data sources are planned.

In conclusion, approximately one quarter less fragility hip fractures were admitted to our hospital during lockdown in Madrid, Spain, compared to control periods in 2018 and 2019. Patients' age, gender, baseline functional and cognitive status and residential status were, however, similar between the periods observed.
Author contributions All authors have contributed to the conception and design, acquisition of data, and analysis and interpretation of data; drafting the article and revising it critically for important intellectual content; and approval of the version to be published and all subsequent versions.

Funding The present study did not receive any external funding.

Data availability Data will be made available upon request to the corresponding author.

\section{Compliance with ethical standards}

Conflict of interest Author COT received grants and honoraria from AMGEN, UCB and MBA Ibérica, none of them related to this work. Aothors JCG and LRRP have no conflicts of interest to declare.

Ethical approval Institutional Review Board approval was obtained for this study, which was classified as "non-interventional" by the Spanish Medicines Agency.

Consent to participate For this type of retrospective study, formal consent is not required according to local legislation.

\section{References}

1. Leavy B, Åberg AC, Melhus H, Mallmin H, Michaëlsson K, Byberg L (2013) When and where do hip fractures occur? A population-based study. Osteoporos Int 24:2387-2396. https:// doi.org/10.1007/s00198-013-2333-6

2. Kanis JA, Odén A, McCloskey EV, Johansson H, Wahl DA, Cooper C et al (2012) A systematic review of hip fracture incidence and probability of fracture worldwide. Osteoporos Int 23:2239-2256. https://doi.org/10.1007/s00198-012-1964-3

3. Scott CEH, Holland G, Powell-Bowns MFR, Brennan CM, Gillespie M, Mackenzie SP et al (2020) Population mobility and adult orthopaedic trauma services during the COVID-19 pandemic: fragility fracture provision remains a priority. Bone Jt Open 1:182-189. https://doi.org/10.1302/2046-3758.16.BJO2020-0043.R1

4. Benazzo F, Rossi SMP, Maniscalco P, Moretti B, Vaienti E, Ruggieri P et al (2020) The orthopaedic and traumatology scenario during Covid-19 outbreak in Italy: chronicles of a silent war. Int Orthop. https://doi.org/10.1007/s00264-020-04637-3

5. Maniscalco P, Poggiali E, Quattrini F, Ciatti C, Magnacavallo A, Vercelli A et al (2020) Proximal femur fractures in COVID19 emergency: the experience of two Orthopedics and Traumatology Departments in the first eight weeks of the Italian epidemic. Acta Biomed 91:89-96. https://doi.org/10.23750/ abm.v91i2.9636

6. D'Adamo H, Yoshikawa T, Ouslander JG (2020) Coronavirus disease 2019 in geriatrics and long-term care: the ABCDs of COVID-19. J Am Geriatr Soc 68:912-917. https://doi. org/10.1111/jgs. 16445

7. Comas-Herrera A, Zalakaín J, Litwin C, Hsu AT, Lemmon E, Henderson D, et al. Mortality associated with COVID-19 outbreaks in care homes: early international evidence n.d.:28

8. Bello X, Pardo-Seco J, del Molino-Bernal MLP, Martinón-Torres F, Salas A (2020) Phylogeography of SARS-CoV-2 pandemic in Spain: a story of multiple introductions, micro-geographic stratification, founder effects, and super-spreaders. Zool Res 41:1-16 
9. Condes E, Arribas JR (2020) Impact of COVID-19 on Madrid hospital system. Enferm Infecc Microbiol Clin. https://doi. org/10.1016/j.eimc.2020.06.005

10. Sáez-López P, González-Montalvo JI, Ojeda-Thies C, MoraFernández J, Muñoz-Pascual A, Cancio JM et al (2018) Spanish National Hip Fracture Registry (SNHFR): a description of its objectives, methodology and implementation. Rev Esp Geriatr Gerontol 53:188-195. https://doi.org/10.1016/j.regg.2017.12.001

11. Katz S (1983) Assessing self-maintenance: activities of daily living, mobility, and instrumental activities of daily living. J Am Geriatr Soc 31:721-727. https://doi.org/10.1111/j.1532-5415.1983. tb03391.x

12. Pfeiffer E (1975) A short portable mental status questionnaire for the assessment of organic brain deficit in elderly patients. J Am Geriatr Soc 23:433-441

13. Wolters U, Wolf T, Stützer H, Schröder T (1996) ASA classification and perioperative variables as predictors of postoperative outcome. Br J Anaesth 77:217-222

14. COVID-19 Community Mobility Report. COVID-19 Community Mobility Report n.d. https://www.google.com/covid19/mobil ity?hl=es. Accessed July 14, 2020

15. Jansen FM, Prins RG, Etman A, van der Ploeg HP, de Vries SI, van Lenthe FJ et al (2015) Physical activity in non-frail and frail older adults. PLoS ONE 10:e0123168. https://doi.org/10.1371/ journal.pone. 0123168

16. Ajayi B, Trompeter A, Arnander M, Sedgwick P, Lui DF (2020) 40 days and 40 nights: clinical characteristics of major trauma and orthopaedic injury comparing the incubation and lockdown phases of COVID-19 infection. Bone Jt Open 1:330-338. https:// doi.org/10.1302/2046-3758.17.BJO-2020-0068.R1

17. Park C, Sugand K, Nathwani D, Bhattacharya R, Sarraf KM (2020) Impact of the COVID-19 pandemic on orthopedic trauma workload in a London level 1 trauma center: the "golden month." Acta Orthop. https://doi.org/10.1080/17453674.2020.1783621

18. Stoker S, McDaniel D, Crean T, Maddox J, Jawanda G, Krentz $\mathrm{N}$ et al (2020) The effect of shelter-in-place orders and the COVID-19 pandemic on orthopaedic trauma at a community level II trauma center. J Orthop Trauma. https://doi.org/10.1097/ BOT.0000000000001860

19. Fahy S, Moore J, Kelly M, Flannery O, Kenny P (2020) Analysing the variation in volume and nature of trauma presentations during COVID-19 lockdown in Ireland. Bone Jt Open 1:261-266. https ://doi.org/10.1302/2046-3758.16.BJO-2020-0040.R1
20. Hernigou J, Morel X, Callewier A, Bath O, Hernigou P (2020) Staying home during "COVID-19" decreased fractures, but trauma did not quarantine in one hundred and twelve adults and twenty eight children and the "tsunami of recommendations" could not lockdown twelve elective operations. Int Orthop. https ://doi.org/10.1007/s00264-020-04619-5

21. Nuñez JH, Sallent A, Lakhani K, Guerra-Farfan E, Vidal N, Ekhtiari S et al (2020) Impact of the COVID-19 pandemic on an emergency traumatology service: experience at a tertiary trauma centre in Spain. Injury 51:1414-1418. https://doi.org/10.1016/j. injury.2020.05.016

22. Lv H, Zhang Q, Yin Y, Zhu Y, Wang J, Hou Z et al (2020) Epidemiologic characteristics of traumatic fractures during the outbreak of coronavirus disease 2019 (COVID-19) in China: a retrospective \& comparative multi-center study. Injury. https://doi. org/10.1016/j.injury.2020.06.022

23. Zhu Y, Chen W, Xin X, Yin Y, Hu J, Lv H et al (2020) Epidemiologic characteristics of traumatic fractures in elderly patients during the outbreak of coronavirus disease 2019 in China. Int Orthop. https://doi.org/10.1007/s00264-020-04575-0

24. Hall AJ, Clement ND, Farrow L, MacLullich AMJ, Dall GF, Scott CEH et al (2020) IMPACT-Scot report on COVID-19 and hip fractures. Bone Joint J 102-B:1219-1228. https://doi. org/10.1302/0301-620X.102B9.BJJ-2020-1100.R1

25. Muñoz Vives JM, Jornet-Gibert M, Cámara-Cabrera J, Esteban PL, Brunet L, Delgado-Flores L et al (2020) Mortality rates of patients with proximal femoral fracture in a worldwide pandemic: preliminary results of the Spanish HIP-COVID observational study. J Bone Joint Surg Am 102:e69. https://doi.org/10.2106/ JBJS.20.00686

26. Egol KA, Konda SR, Bird ML, Dedhia N, Landes EK, Ranson RA et al (2020) Increased mortality and major complications in hip fracture care during the COVID-19 pandemic: a New York City perspective. J Orthop Trauma 34:395-402. https://doi. org/10.1097/BOT.0000000000001845

Publisher's Note Springer Nature remains neutral with regard to jurisdictional claims in published maps and institutional affiliations. 